\title{
DENSIDAD DE NIDOS DE LA COMUNIDAD DE HORMIGAS (FORMICIDAE) EN TRES OLIVARES CON DIFERENTE MANEJO AGRONÓMICO EN GRANADA, ESPAÑA
}

\section{NEST DENSITY OF THE ANT ASSEMBLAGE (FORMICIDAE) IN THREE OLIVE ORCHARDS UNDER DIFFERENT AGRICULTURAL MANAGEMENT IN GRANADA, SPAIN}

\author{
Inés Redolfi ${ }^{1}$, Alberto Tinaut ${ }^{2}$, Felipe Pascual $^{2}$ y Mercedes Campos ${ }^{3}$
}

\section{Resumen}

Se han estudiado los nidos de hormigas presentes en tres olivares con diferente manejo agronómico, ubicados a $20 \mathrm{~km}$ de la ciudad de Granada (España). En cada olivar se observó el área correspondiente a 60 árboles (seis hileras contiguas de 10 árboles) y los cinco transectos de $100 \mathrm{~m}$ de longitud por un metro de ancho en las calles, entre las hileras de árboles. En el suelo bajo la copa de cada árbol y en las calles se determinó el número de los nidos en actividad de cada especie, en un área total de $540 \mathrm{~m}^{2}\left(9 \mathrm{~m}^{2} /\right.$ árbol) y $500 \mathrm{~m}^{2}\left(100 \mathrm{~m}^{2} /\right.$ calle $)$ respectivamente, con una duración de 15 minutos por árbol y por transecto entre las 9:00 y 14:00 horas. También se determinó el número de nidos en el tronco del árbol mediante observación de los mismos en 5 minutos, durante los meses de Mayo, Julio y Septiembre de 1997 y 1998. Los resultados indican que la perturbación producida por las prácticas agronómicas afecta el número y abundancia de los hormigueros en el suelo bajo el árbol y en las calles. Los olivares con manejo ecológico (Arenales) y abandonado (Colomera 2) presentaron significativamente mayor densidad de nidos en el suelo bajo el árbol que el olivar con aplicación de plaguicidas (Colomera 1). Este último no presentó hormigueros en las calles. La perturbación por laboreo y aplicación de plaguicidas en el olivar abandonado tuvo como respuesta el aumento de nidos por gemación y la disminución posterior de los mismos. En los árboles de 80 años (Arenales) se presentan hormigueros en el tronco y no en los de 25 años.

Palabras claves: Formicidae, densidad de nidos, olivar, diferente manejo agronómico, Granada, España.

\begin{abstract}
Ant nests were studied in three olive orchards under different agricultural management located $20 \mathrm{~km}$ away from Granada City, Spain. In each orchard, It was monitored the area covered by 60 trees (six contiguous rows of 10 trees each) and five transects of $100 \mathrm{~m}$ long and $1 \mathrm{~m}$ wide on the alleyways among the tree rows. It was determined for each ant specie, the number of active nests in the ground under each tree crown $-9 \mathrm{~m}^{2} /$ tree- and in the alleyways $-100 \mathrm{~m}^{2} /$ alley- for a period of 15 min per tree and per transect between 09:00 and 14:00 hours, during May, July and September of 1997 and 1998. It was also determined the number of ant nests within the tree trunk through five-minute observation intervals. Results indicate that disturbances caused by cultural practices affect the number and abundance of ant nests in the ground under the trees and in the alleys. Olive orchards with an ecological management (Arenales) or abandoned (Colomera 2) showed significantly higher nest density in the ground below the tree than the orchard with pesticide application (Colomera 1). This orchard did not show any nest in the alleys. Cultural practices and pesticide applications done in the abandoned olive orchard resulted in the increase of subsidiary nests and their posterior decrease. Eighty-year old olive trees (Arenales) show nests in their trunks, while 25-year old trees do not show them.
\end{abstract}

Keys words: Formicidae, nests density, olive orchards, different agricultural management, Granada, España.

\section{Introducción}

Las hormigas ocupan un lugar muy importante en los ecosistemas, interviniendo en el ciclo de nutrientes en la naturaleza, en el enriquecimiento de los suelos y en una gran diversidad de interacciones tróficas, de tal manera que son mencionadas como controladores biológicos de plagas, bioindicadores y como elementos importantes para definir el índice de biodiversidad integral (Adlung, 1966; Majer, 1972,
1994; Andersen, 1990; Majer \& Beeston, 1996; Detraint, 1990; Paulson \& Akre, 1992; Fiala et al., 1994; Weseloch, 1993, 1996; Peng et al., 1997; Rabistch, 1997; Morris, 1997; Redolfi, 1999; Morris et al., 1998a; Morris et al., 2002).

Las hormigas forman parte de la estructura y función del agrosistema del olivo (Olea europeae L.) y los estudios de la biocenosis se han intensificado en este cultivo, especialmente frente a la demanda de cultivos 
ecológicos (Morris, 1997; Morris et al., 1998a b; Redolfi et al., 2003). Así, el estudio para determinar el número $\mathrm{y}$ densidad de los hormigueros de la comunidad de hormigas en olivares con diferentes prácticas agronómicas, contribuirá a generar conocimiento en la línea base, para utilizar a las hormigas como bioindicadores para el manejo de este agrosistema.

\section{Materiales y Métodos \\ Ubicación y vegetación potencial de la zona de estudio}

La zona de estudio se encuentra a $20 \mathrm{~km}$ al norte de la ciudad de Granada (Andalucía). La vegetación se corresponde con un encinar más o menos sustituido por el cultivo del olivo. La mayor parte del olivar se sitúa en los pisos Termo y Mesomediterráneo en los cuales la vegetación climácica se correspondería con la serie de los acebuchales, para el piso Termomediterráneo y de los encinares para el piso Mesomediterráneo (Rivas-Martínez, 1987). En el primitivo bosque existían especies típicas del clima de la Meseta Mediterránea, como la encina (Quercus ilex L.), o el pino de alepo (Pinus alepensis Miller) y según lo permitiera la constitución edáfica, el alcornoque (Quercus suber L.); alternando con especies herbáceas como Smilax aspera L., Rhamnus alaternus L., Daphne gnidium L., Helleborus foetidus L. y Paeonia broteri Boiss. \& Reut., entre otras. La vegetación primitiva de la zona puede todavía contemplarse en los linderos y claros del olivar. Por degradación, se ha producido una vegetación arbustiva que comprende, entre otras, a Asparagus acutifolius L., Convolvulus arvensis L., Euphorbia characias L., Reseda lutea L. y Rubus ulmifolius Schoot. Sin embargo, la mayoría son especies de flora arvense más o menos ruderal y de amplia distribución geográfica (González, 1989; Morris, 1997).

\section{Características de los tres olivares}

Las observaciones se realizaron en tres olivares: Arenales, Colomera 1 y Colomera 2, plantados a marco real con distancia entre pies de unos $10 \mathrm{~m} \mathrm{y}$ altura media de $3.5 \mathrm{~m}$. El arado de las zonas claras del olivar (calles), forma un cuadrado sin arar debajo de la copa. Debido a esta práctica, se puede considerar que el olivar presenta un diseño muy característico en comparación con otros cultivos: los árboles en el centro de un cuadrado de unos $9 \mathrm{~m}^{2}$ sin arar, separados entre sí por calles aradas de $7 \mathrm{~m}$, prácticamente sin vegetación. Los tres olivares se diferencian por las prácticas agronómicas: Arenales. El cortijo "Arenales de San Pedro" abarca una extensión de 525 ha con un total aproximado de 28600 árboles. Los olivos tienen una edad de 80 años y son de tres pies. El riego es por inundación y el laboreo de tipo tradicional. En 1997 se llevó a cabo una poda severa, mientras que en 1998 sólo un aclareo. No se realizaron tratamientos con agroquímicos. El suelo es franco arenoso (Barahona, com. per.), con un porcentaje de grava del $43.97 \%$ bajo el árbol y del $34.49 \%$ en las calles. Los bancales, con escasa vegetación arbustiva están distribuidos entre las parcelas y en los límites del cultivo. Colomera. La finca "La Granja" tiene una extensión de 320 ha con aproximadamente 16300 árboles. Los olivos tienen una edad de 25 años y son de dos y tres pies. En este olivar se escogieron dos parcelas separadas por una distancia de $600 \mathrm{~m}$. Colomera 1: El riego es por goteo de cuatro mangueras por árbol, con fertirrigación y el laboreo correspondiente a un olivar de tipo moderno. El tipo de poda fue similar a la de Arenales. Se hicieron tratamientos con agroquímicos contra Pyays oleae Bern. y Batrocera oleae (Gmel.) y herbicidas bajo la copa del árbol. Presenta un suelo franco bajo el árbol y franco-arcilloso en las calles (Barahona, com.per), con un porcentaje de grava del $40.46 \%$ bajo la copa del árbol y del $32.13 \%$ en las calles. Los bancales se encuentran sólo en ecasas áreas limítrofes del olivar. Colomera 2: Es una parcela de 1ha, abandonada durante 10 años, tiempo en el cual no se efectuó ningún tipo de laboreo, riego o tratamiento fitosanitario. Está rodeada por el olivar con riego por goteo a manera de una pequeña isla. Este olivar no presentó al inicio del estudio el cuadrado de área sin arar bajo la copa del árbol, ya que hasta Julio de 1997 todo el suelo de la zona estaba cubierto por malezas y después de esta fecha, el arado severo fue hasta el pie del árbol, eliminando todo tipo de vegetación y en 1998 se prosiguió con esta labor y se realizaron tratamientos con agroquímicos. Presenta un suelo franco (Barahona, com.per.), con un porcentaje de grava del $44.34 \%$ bajo el árbol y del $42.96 \%$ en las calles.

\section{Variedad de olivo}

Las variedades de olivo que se encuentran cultivadas en la zona de estudio son "picual" o "marteño" en más del $80 \%$ y "hojiblanco" en un $15 \%$. Las observaciones del presente trabajo se realizaron sobre la variedad "picual", también denominada como "nevadillo" y "lopereño", que es la principal variedad distribuida en las zonas de Córdoba, Jaén y Granada.

\section{Factores abióticos}

El clima se caracteriza por inviernos fríos, así como por un largo periodo estivo-otoñal de elevadas temperaturas y muy seco. La pluviometría media anual es de unos $500 \mathrm{~mm}$, con reparto normalmente igualado, excepto de Junio a Agosto donde es muy escasa. La humedad relativa no presenta por lo general valores muy elevados (38.5-65\%), salvo en ciertas fechas muy determinadas. La climatología, típicamente continental, produce importantes variaciones térmicas y pluviométricas. Las heladas suelen presentarse de Noviembre a Marzo, ambos inclusive, aunque no es raro su presencia fuera de dicha época. Generalmente, las temperaturas máximas alcanzan sus valores más elevados $\left(43^{\circ} \mathrm{C}-45^{\circ} \mathrm{C}\right)$ en Junio-Agosto, lo que, en principio puede constituir un 
factor de gran importancia en el condicionamiento del cultivo y de los fitófagos (Morris, 1997). El suelo: corresponde a un tipo ambisol cálcico sobre costra caliza de buen drenaje con escaso abonado orgánico (Pérez \& Prieto, 1980). Está formado sobre sedimentos del Plioceno, profundos, bien drenados, de textura franca y muy aptos para el cultivo del olivar. El nitrógeno es un elemeno deficitario, que se debe administrar con la fertilización. El contenido de K del suelo es aceptable, si bien con frecuencia se observan síntomas de deficiencia de este elemento en el cultivo, por interacción con la absorción con el calcio. Normalmente, el suelo presenta escaso abonado mineral y es de secano (González, 1989).

Los datos climatológicos correspondientes a los años de estudio 1997 y 1998, se presentan en Redolfi (1999) y Redolfi et al. (1999, 2003). Así mismo, estos autores determinan que no existen diferencias significativas en la temperatura, humedad y precipitación en las tres zonas de estudio, en cada fecha de muestreo.

\section{Determinación del número de hormigueros}

En cada olivar se observó el área correspondiente a 60 árboles (seis hileras contiguas de 10 árboles) y los cinco transectos de $100 \mathrm{~m}$ de longitud por un metro de ancho en las calles, entre las hileras de árboles. En el suelo correspondiente a cada árbol y en las calles, en un área que supone un total de $540 \mathrm{~m}^{2}$ ( $9 \mathrm{~m}^{2} /$ árbol) y $500 \mathrm{~m}^{2}\left(100 \mathrm{~m}^{2} /\right.$ calle $)$ respectivamente, se determinó el número de los nidos en actividad de cada especie. La observación y toma de muestras tuvo una duración de 15 minutos por árbol y por transecto entre las 9:00 y 14:00 hs, en los meses de Mayo, Julio y Septiembre de 1997 y 1998. Esta metodología ha sido ampliamente utilizada tanto en ecosistemas naturales (Bernstein \& Gobbel, 1979; Tinaut, 1979; Cushman et al., 1988; Cerdá \& Retana, 1988; Dejean et al., 1994; Orr \& Charles, 1994; Ofer et al., 1996; Cerdá \& Retana, 1998), como también en agroecosistemas arbóreos, principalmente de cacao, café, mango y palmera, para realizar el mapeo de las diferentes especies (Room, 1971, 1974; Majer, 1972, 1987, 1994; Leston, 1978; Jackson, 1984; Majer \& Delabie, 1993; Perfecto \& Snelling, 1995; Peng et al., 1997).

\section{Análisis de los datos}

El análisis de las variables entre los diferentes meses, años y zonas de muestreo se efectuó mediante el test no-paramétrico de suma de rangos de KruskalWallis. Así también, se utlilizó el test no-paramétrico $\mathrm{U}$ de Mann-Whitney y las pruebas estadísticas correspondientes del paquete estadístico Stat View y Systat 5.0 para Macintosh.

\section{Resultados y discusión}

Número de nidos en el suelo bajo la copa del árbol

Se presentaron 14 especies con nidos en el suelo, bajo la copa del árbol: Aphaenogaster gibbosa (Latreille), A. senilis Mayr, Messor barbarus (L.), Crematogaster auberti Emery, C. sordidula (Nylander), Solenopsis latro Forel, Tetramorium semilaeve André, Tapinoma nigerrima (Nylander), Plagiolepis pygmaea (Latreille), Camponotus foreli Emery, C. sylvaticus Olivier, Cataglyphis rosenhaueri Santschi, C. velox Santschi y Formica subrufa Roger.

Tabla 1. Número de nidos en el suelo bajo la copa del árbol $(n=60)$ en el olivar Arenales. 1997.

\begin{tabular}{lccc}
\hline Especie & Mayo & Julio & Septiembre \\
\hline A. gibbosa & 2 & 1 & 0 \\
A. senilis & 16 & 23 & 5 \\
M. barbarus & 0 & 1 & 0 \\
C. sordidula & 1 & 0 & 7 \\
S. latro & 2 & 0 & 10 \\
T. semilaeve & 3 & 2 & 6 \\
T. nigerrima & 8 & 8 & 3 \\
$P$. pygmaea & 24 & 20 & 1 \\
C. foreli & 1 & 0 & 0 \\
C. sylvaticus & 2 & 2 & 2 \\
C. rosenhaueri & 1 & 2 & 2 \\
F. subrufa & 1 & 0 & 0 \\
\hline Total & 61 & 59 & 36 \\
\hline
\end{tabular}

Arenales: manejo ecológico

El mayor número de especies correspondió al olivar Arenales $(\mathrm{S}=13)$, a continuación Colomera $2(\mathrm{~S}=8)$ y Colomera $1(\mathrm{~S}=5)$. Las especies comunes a las tres zonas fueron: $M$. barbarus, $T$. semilaeve, $T$. nigerrima, $P$. pygmaea y $C$. rosenhaueri. Las especies T. nigerrima, $P$. pygmaea y $A$. senilis constituyeron el mayor número de nidos $\mathrm{y}$ permanecieron activos durante toda la campaña. A. senilis sólo estuvo presente en el olivar de Arenales (Tablas 1-6). La ubicación de la entrada de los hormigueros fue de manera definida para la mayoría de las especies (Redolfi et al., 1999). En el olivar de Arenales, el número de nidos en el suelo más frecuente por árbol durante toda la campaña fue de dos $(n=21)$ y tres $(n=9)$. En los olivares Colomera 1 y Colomera 2, se detectó generalmente un nido en el suelo bajo la copa del árbol, a excepción del muestreo del mes de Septiembre en Colomera 2, en que se encontraron con mayor frecuencia dos nidos por árbol $(\mathrm{n}=18)$ debido al aumento de las poblaciones de $T$. semilaeve y $S$. latro. Estas dos especies se encontraron en el suelo bajo la copa del mismo árbol, pero con un comportamiento agresivo de $T$. semilaeve, cuyas obreras rodeaban los frutos de aceituna en donde se encontraban refugiadas las obreras de S. latro (Redolfi et al., 1999). La 
presencia conjunta de especies con nidos en el suelo bajo la copa del árbol implicó a la mayoría de las especies en este habitat. Las frecuencias mayores correspondieron a las especies $A$. senilis-P. pygmaea $(\mathrm{n}=9)$ y $A$. senilis- $T$. nigerrima $(\mathrm{n}=5)$. En todos estos casos no se observó un comportamienteo agresivo entre las obreras de diferentes especies, lo cual no coincide con la agresividad reportada para $T$. nigerrima (Cerdá et al., 1989). Las únicas especies que no compartieron el suelo bajo la copa del árbol fueron M. barbarus, C. foreli y F. subrufa.

Tabla 2. Número de nidos en el suelo bajo la copa del árbol $(n=60)$ en el olivar Colomera 1. 1997.

\begin{tabular}{lccc}
\hline Especie & Mayo & Julio & Septiembre \\
\hline M. barbarus & 1 & 0 & 0 \\
T. semilaeve & 2 & 0 & 0 \\
T. nigerrima & 5 & 2 & 3 \\
P. pygmaea & 5 & 7 & 0 \\
\hline Total & 13 & 9 & 3 \\
\hline
\end{tabular}

Colomera 1: manejo con pesticidas

Tabla 3. Número de nidos en el suelo bajo la copa del árbol $(n=60)$ en el olivar Colomera 2. 1997.

\begin{tabular}{lccc}
\hline Especie & Mayo & Julio & Septiembre \\
\hline M. barbarus & 0 & 4 & 6 \\
C. auberti & 14 & 10 & 0 \\
S. latro & 0 & 0 & 12 \\
T. semilaeve & 0 & 0 & 58 \\
T. nigerrima & 46 & 9 & 1 \\
$P$. pygmaea & 1 & 0 & 0 \\
C. rosenhaueri & 0 & 1 & 0 \\
\hline Total & 61 & 24 & 77 \\
\hline Co.lomera $2:$ aband
\end{tabular}

Colomera 2: abandonado. Arado severo a partir de Julio 1997.

Los olivares Colomera 2 y Arenales, presentaron el mayor número de nidos en 1997, con diferencias altamente significativas $(\mathrm{P}<0.0001)$ al olivar Colomera 1 , debido probablemente a la aplicación de agroquímicos en este último, tal como mencionan Buren \& Whitcomb (1997) con la disminución de hormigas en cultivos de cítricos por aplicación de plaguicidas (Tablas 1-3, 7 y 11).

El número de nidos fue superior durante 1997 con respecto a 1998 en las tres zonas (significativamente, $\mathrm{P}<0.0001$ en Arenales y Colomera 2; no significativa, $\mathrm{P}=0.1$ en Colomera 1) (Tabla 7), debido probablemente a las diferencias climáticas entre los dos años (Redolfi et al., 2003), lo cual puede haber influido de manera directa sobre el comportamiento de las diferentes especies de hormigas o bien de forma indirecta al afectar la fenología del árbol y malas hierbas que representan la base del nicho de los Formicidae. En el año 1998 se observó un descenso

Tabla 4. Número de nidos en el suelo bajo la copa del árbol $(\mathrm{n}=60)$ en el olivar Arenales. 1998.

\begin{tabular}{lccc}
\hline Especie & Mayo & Julio & Septiembre \\
\hline A. gibbosa & 1 & 1 & 0 \\
A. senilis & 20 & 22 & 2 \\
A. auberti & 1 & 0 & 0 \\
C. sordidula & 0 & 1 & 0 \\
S. latro & 0 & 0 & 4 \\
T. semilaeve & 0 & 0 & 6 \\
T. nigerrima & 11 & 2 & 0 \\
P. pygmaea & 10 & 2 & 0 \\
C. sylvaticus & 3 & 1 & 0 \\
\hline Total & 46 & 29 & 12 \\
\hline
\end{tabular}

Tabla 5. Número de nidos en el suelo bajo la copa del árbol $(n=60)$ en el olivar Colomera 1.1998.

Especie Mayo Julio Septiembre

\begin{tabular}{llll}
$M$. barbarus & 1 & 0 & 0 \\
$T$. nigerrima & 3 & 2 & 0 \\
$P$. pygmaea & 2 & 0 & 0 \\
C. rosenhaueri & 0 & 0 & 1 \\
\hline Total & 6 & 2 & 1 \\
\hline
\end{tabular}

de las precipitaciones $y$ un aumento de las temperaturas a partir del mes de Abril, con respecto a 1997. Esto ocasionó una disminución del período de floración del árbol y las malezas, y provocó el marchitamiento de la cubierta vegetal del suelo en el mes de Julio, con una antelación de aproximadamente 25 días, con respecto al año 1997. Estas diferencias climáticas entre los dos años, explicarían la disminución tan marcada del número de nidos de $P$. pygmaea en Arenales y en menor grado en Colomera 1, considerando que esta especie resiste con dificultad las temperaturas elevadas (Retana et al., 1989).

Tabla 6. Número de nidos en el suelo bajo la copa del árbol $(n=60)$ en el olivar Colomera 2. 1998.

\begin{tabular}{lccc}
\hline Especie & Mayo & Julio & Septiembre \\
\hline M. barbarus & 0 & 0 & 4 \\
T. nigerrima & 11 & 2 & 0 \\
C. rosenhaueri & 0 & 1 & 0 \\
C. velox & 1 & 0 & 0 \\
\hline Total & 12 & 3 & 4 \\
\hline
\end{tabular}

Colomera 2: manejo con pesticidas en 1998

Asímismo, estas condiciones pudieron ser las causantes de la desaparición de $T$. nigerrima a partir del mes de Julio de 1998 en los tres olivares. Sin 
embargo, en Colomera 2, la disminución altamente significativa $(\mathrm{P}<0.001)$ del número de nidos tiene otra explicación aparte de las diferencias climáticas entre ambos años, ya que el laboreo intenso iniciado en este cultivo en Julio de 1997 y las aplicaciones de plaguicidas realizadas en 1998, provocaron probablemente la disminución del número de nidos bajo la copa del árbol y esta zona pasó a ser un continuo con el olivar que lo rodeaba (Tablas 1-7 y 11).

Tabla 7. Valores medios, mínimos y máximos de nidos en el suelo/árbol $(n=60)$, en los tres olivares. 1997 y 1998.

\begin{tabular}{|c|c|c|c|c|c|}
\hline \multirow[b]{2}{*}{ Zona } & \multirow[b]{2}{*}{ Año } & \multicolumn{4}{|c|}{ Número de nidos } \\
\hline & & Mes & $\bar{x} \pm s$ & Mín. & Máx. \\
\hline \multirow[t]{3}{*}{ Arenales } & 1997 & Mayo & $1.03 \pm 1.17 \mathrm{a} \mathrm{df}$ & 0.00 & 4.00 \\
\hline & & Julio & $0.95 \pm 0.79 \mathrm{a}$ & 0.00 & 3.00 \\
\hline & & Sept. & $0.60 \pm 0.78 b$ & 0.00 & 3.00 \\
\hline \multirow[t]{3}{*}{ Colomera 1} & & Mayo & $0.21 \pm 0.45$ a ef & 0.00 & 2.00 \\
\hline & & Julio & $0.15 \pm 0.40 \mathrm{a}$ & 0.00 & 2.00 \\
\hline & & Sept. & $0.05 \pm 0.22 \mathrm{a}$ & 0.00 & 1.00 \\
\hline \multirow[t]{3}{*}{ Colomera 2} & & Mayo & $1.01 \pm 0.59 \mathrm{a} \mathrm{d} \mathrm{f}$ & 0.00 & 2.00 \\
\hline & & Julio & $0.41 \pm 0.61 \mathrm{~b}$ & 0.00 & 2.00 \\
\hline & & Sept. & $1.28 \pm 0.58 \mathrm{c}$ & 0.00 & 3.00 \\
\hline \multirow[t]{3}{*}{ Arenales } & 1998 & Mayo & $0.75 \pm 0.87$ a d g & 0.00 & 3.00 \\
\hline & & Julio & $0.48 \pm 0.53 \mathrm{a}$ & 0.00 & 2.00 \\
\hline & & Sept. & $0.20 \pm 054 \mathrm{~b}$ & 0.00 & 3.00 \\
\hline \multirow[t]{3}{*}{ Colomera 1} & & Mayo & $0.10 \pm 0.30$ a ef & 0.00 & 1.00 \\
\hline & & Julio & $0.03 \pm 0.18 \mathrm{a}$ & 0.00 & 1.00 \\
\hline & & Sept. & $0.01 \pm 0.12 \mathrm{a}$ & 0.00 & 1.00 \\
\hline \multirow[t]{3}{*}{ Colomera 2} & & Mayo & $0.20 \pm 0.40$ a e g & 0.00 & 1.00 \\
\hline & & Julio & $0.05 \pm 022 \mathrm{~b}$ & 0.00 & 1.00 \\
\hline & & Sept. & $0.08 \pm 0.33 \mathrm{~b}$ & 0.00 & 2.00 \\
\hline
\end{tabular}

Las cifras con la misma letra no difieren significativamente entre sí (Kruskal-Wallis y la prueba de la U de Mann-Whitney, $\mathrm{P}>0.05)$.

a, b, c: entre meses en cada zona;

d, e: entre zonas en cada año;

$f$, $g$ : entre años en cada zona.

El número de nidos tuvo una tendencia a disminuir entre los meses de Mayo a Septiembre, en ambos años, en las tres zonas (Tablas 1-6). En Arenales las diferencias no fueron significativas entre los meses de Mayo y Julio (1997: $\mathrm{P}=0.6 ; 1998: \mathrm{P}=0.2$ ), pero si lo fueron entre Julio y Septiembre $(\mathrm{P}<0.05$, ambos años). Colomera 1 no presentó deferencias significativas entre meses en los dos años $(\mathrm{P}>0.05)$ y Colomera 2 se caracterizó por diferencias altamente significativas entre meses $(\mathrm{P}<0.0001)$ en el año 1997 y la ausencia de diferencias significativas entre los meses de Julio y Septiembre $(\mathrm{P}>0.05)$ en 1998 , debido al laboreo que se inició en esta zona (Tabla 7). Sin embargo, en el olivar Colomera 2, el número de nidos aumentó significativamente $(\mathrm{P}<0.001)$ en el mes de Septiembre de 1997 debido a la aparición de $T$. semilaeve, lo cual también ocurrió en Arenales, aunque en menor grado, mientras que en Colomera 1 esta especie no se presentó (Tablas 1-6). Este aumento de la población de $T$. semialeve y aparición de $S$. latro no se vuelve a producir en el olivar Colomera 2 en 1998, debido probablemente al uso de plaguicidas durante ese año, mientras que en Arenales se presentaron nuevamente las dos especies, lo cual indica que la ausencia de los dos especies en Colomera 2 no se debe a los cambios en las condiciones climáticas (Tablas 4 y 6).

Tabla 8. Número de nidos en las calles en los olivares Arenales y Colomera 2. 1997.

\begin{tabular}{|c|c|c|c|c|}
\hline Zona & Especie & Мayo & Julio & Septiembre \\
\hline \multirow[t]{5}{*}{ Arenales } & A. senilis & 0 & 2 & 0 \\
\hline & M. barbarus & 0 & 1 & 0 \\
\hline & C. sylvaticus & 0 & 1 & 0 \\
\hline & C. rosenhaueri & 1 & 0 & 0 \\
\hline & Total & 1 & 4 & 0 \\
\hline \multirow[t]{6}{*}{ Colomera 2} & M. barbarus & 13 & 10 & 46 \\
\hline & M. bouvieri & 0 & 1 & 16 \\
\hline & T. nigerrima & 7 & 7 & 12 \\
\hline & C. rosenhaueri & 5 & 1 & 2 \\
\hline & C. velox & 0 & 1 & 0 \\
\hline & Total & 25 & 20 & 76 \\
\hline
\end{tabular}

\section{Número de nidos en las calles}

Las ocho especies con hormigueros en las calles del olivar fueron: A. senilis, M. barbarus, Messor bouvieri Bondroit, T. nigerrima, C. sylvaticus, $C$. rosenhaueri, $C$. velox y $F$. subrufa. La especie $M$. bouvieri fue la única especie que presentó nidos exclusivamente en las calles del olivar. En el olivar de Arenales se observó mayor número de especies $(n=6)$ que en Colomera $2(\mathrm{n}=5)$, mientras que Colomera 1 no presentó nidos en las calles. Las especies comunes a las dos zonas fueron M. barbarus, C. rosenhaueri y C. velox (Tablas 8-9).

Tabla 9. Número de nidos en las calles en los olivares Arenales y Colomera 2. 1998.

\begin{tabular}{llccc} 
Zona & Especie & Mayo & Julio & Septiembre \\
\hline \multirow{4}{*}{ Arenales } & M. barbarus & 1 & 0 & 0 \\
& C. rosenhaueri & 4 & 1 & 0 \\
& C. velox & 0 & 1 & 0 \\
& F. subrufa & 0 & 1 & 0 \\
\cline { 2 - 5 } Total & 5 & 3 & 0 \\
\cline { 2 - 5 } Colomera & 2M. barbarus & 26 & 10 & 0 \\
& C. rosenhaueri & 8 & 0 & 0 \\
\hline & Total & 34 & 10 & 0
\end{tabular}

En el olivar Colomera 2 el número de nidos fue significativamente más elevado que en Arenales 
$(\mathrm{P}<0.0001)$ en los dos años de estudio, excepto en el mes de Septiembre de 1998 en el cual no se observaron hormigueros en las calles a causa del laboreo que se inicio en esta zona (Tablas 10 y 11). Igualmente, el escaso número de hormigueros en Arenales $(n=1-5)$ que no presentó diferencias significativas $(\mathrm{P}=0.6)$ entre años y entre Mayo y Julio en ambos años $(\mathrm{P}=0.8 ; \mathrm{P}=0.1)$ y la ausencia de estos en Colomera 1 se debió al laboreo continuo en las calles que ocasionó un gran disturbio en el área.

Tabla 10. Valores medios, mínimos y máximos de nidos/ transecto (5 calles) en los tres olivares. $1997 \mathrm{y}$ 1998.

\begin{tabular}{|c|c|c|c|c|c|}
\hline & \multicolumn{5}{|c|}{ Número de nidos } \\
\hline Zona & Año & Mes & $x \pm s$ & Mín. & Máx. \\
\hline \multirow[t]{3}{*}{ Arenales } & 1997 & Mayo & $0.20 \pm 0.44$ a d f & 0.00 & 1.00 \\
\hline & & Julio & $0.80 \pm 0.44 \mathrm{a}$ & 0.00 & 2.00 \\
\hline & & Sept. & $0.00 \quad b$ & 0.00 & 0.00 \\
\hline \multirow[t]{3}{*}{ Colomera 2} & & Mayo & $5.00 \pm 3.00$ a e g & 0.00 & 8.00 \\
\hline & & Julio & $4.00 \pm 2.34 \mathrm{a}$ & 0.00 & 6.00 \\
\hline & & Sept. & $15.00 \pm 2.16 \mathrm{~b}$ & 0.00 & 18.00 \\
\hline \multirow[t]{3}{*}{ Arenales } & 1998 & Mayo & $0.80 \pm 0.83$ a d f & 0.00 & 2.00 \\
\hline & & Julio & $0.60 \pm 0.54 \mathrm{a}$ & 0.00 & 1.00 \\
\hline & & Sept. & $0.00 \quad a$ & 0.00 & 0.00 \\
\hline \multirow[t]{3}{*}{ Colomera 2} & & Mayo & $6.80 \pm 0.83$ a e g & 6.00 & 8.00 \\
\hline & & Julio & $2.00 \pm 1.22 \mathrm{~b}$ & 0.00 & 3.00 \\
\hline & & Sept. & $0.00 \quad \mathrm{c}$ & 0.00 & 0.00 \\
\hline
\end{tabular}

Las cifras con la misma letra no difieren significativamente entre si (Kruskal-Wallis y la prueba de la U. de Mann-Whitney, P>0.05).

$a, b, c$ : entre meses en cada zona

$\mathrm{d}$, e: entre zonas en cada año

$\mathrm{f}, \mathrm{g}$ : entre años en cada zona campaña en 1997, ya que este formicido es muy abundante en terrenos de cultivo abandonados (Acosta, 1980), que permite el establecimiento de malezas cuyas semillas contribuyen a que se mantenga este formícido de costumbres marcadamente granívora. También, se observaron numerosas pistas de esta especie, esto es, rutas principales de forrageo asociadas a la existencia de fenómenos persistentes a una determinada organización de territorio (Oster \& Wilson, 1978). Un hormiguero puede tener una o más pistas simultáneamente (Rosengren, 1977; Cherix \& Rosengren, 1979), cuyo trazado presenta en algunos casos gran estabilidad y persistencia en el tiempo (Rosengren, 1977; David \& Wood, 1980). Estas pistas minimizan los encuentros entre miembros de distintos nidos (Harrison \& Gentry, 1981; Porter \& Jogersen, 1981), evitando así las agresiones que se han observado después de provocar un disturbio en las zonas circundantes a los nidos (Acosta et al., 1985). En Colomera 2, al inicio de los muestreos en Mayo de 1997, todas las calles mostraban una cubierta vegetal continua $y$ se contabilizaron un total de 25 hormigueros pertenecientes a tres especies. Igualmente, Castro et al. (1996), observan una influencia positiva de la cubierta vegetal sobre la abundancia de hormigas. En el mes de Julio se inició el laboreo con un arado profundo que provocó un gran disturbio en toda la zona y se contabilizó 20 hormigueros correspondientes a cinco especies. En esta ocasión se observó el traslado de huevos e inmaduros por parte de las obreras de C. rosenhaueri y $T$. nigerrima, especie que puede cambiar rápidamente su sitio de nidificación por disturbios en el área (Cerdá et al., 1989). El arado continuo de la

Tabla 11. Número $/ \mathrm{m}^{2}$ de hormigueros bajo la copa del árbol y en las calles en los tres olivares. 1997 y 1998.

\begin{tabular}{|c|c|c|c|c|c|c|c|c|c|c|c|c|c|}
\hline \multirow[b]{3}{*}{ Año } & \multirow[b]{3}{*}{ Zona } & \multicolumn{6}{|c|}{ Nidos bajo la copa del árbol } & \multicolumn{6}{|c|}{ Nidos en las calles } \\
\hline & & \multicolumn{2}{|c|}{$\mathrm{M}$} & \multicolumn{2}{|c|}{$\mathrm{J}$} & \multicolumn{2}{|c|}{$\mathrm{S}$} & \multicolumn{2}{|c|}{$\mathrm{M}$} & \multicolumn{2}{|c|}{$\mathrm{J}$} & \multicolumn{2}{|c|}{$\mathrm{S}$} \\
\hline & & $\mathrm{n}$ & $/ \mathrm{m}^{2}$ & $\mathrm{n}$ & $/ \mathrm{m}^{2}$ & $\mathrm{n}$ & $/ \mathrm{m}^{2}$ & $\mathrm{n}$ & $/ \mathrm{m}^{2}$ & $\mathrm{n}$ & $/ \mathrm{m}^{2}$ & $\mathrm{n}$ & $/ \mathrm{m}^{2}$ \\
\hline \multirow[t]{3}{*}{1997} & Arenales & 61 & 0.113 & 59 & 0.109 & 36 & 0.067 & 01 & 0.002 & 04 & 0.007 & 00 & 0.000 \\
\hline & Colomera 1 & 13 & 0.024 & 09 & 0.017 & 03 & 0.006 & 00 & 0.000 & 00 & 0.000 & 00 & 0.000 \\
\hline & Colomera 2 & 61 & 0.113 & 24 & 0.044 & 77 & 0.143 & 25 & 0.046 & 20 & 0.037 & 76 & 0.141 \\
\hline \multirow[t]{3}{*}{1998} & Arenales & 46 & 0.085 & 29 & 0.054 & 12 & 0.022 & 05 & 0.009 & 03 & 0.006 & 00 & 0.000 \\
\hline & Colomera 1 & 06 & 0.011 & 02 & 0.004 & 01 & 0.002 & 00 & 0.000 & 00 & 0.000 & 00 & 0.000 \\
\hline & Colomera 2 & 12 & 0.022 & 03 & 0.006 & 04 & 0.007 & 34 & 0.063 & 10 & 0.019 & 00 & 0.000 \\
\hline
\end{tabular}

M: Mayo, J: Julio, S: Septiembre

En el olivar Colomera 2, con un gran número de nidos ( $\mathrm{n}=20-76)$ durante la campaña 1997, disminuyó el número de hormigueros $(n=10-34)$ y de especies $(\mathrm{n}=2)$ en $1998 \quad(\mathrm{P}=0.008)$. Así, las diferencias altamente significativas entre Colomera 2 y Arenales en $1997(\mathrm{P}<0.0001)$ descienden en 1998 a $\mathrm{P}=0.04$ debido al laboreo que se inició en esta zona (Tabla 10). La especie $M$. barbarus presentó el mayor número de nidos y permaneció durante toda la zona eliminó todas las malezas de las calles e incluso bajo la copa del árbol, pero una vez que cesó el disturbio las hormigas reorganizaron sus nidos y el número se elevó a 76 hormigueros en el mes de Septiembre. $M$. barbarus siguió presentando el mayor número de nidos y se observó en el cono de entrada a los nidos un gran número de restos de obreras de la misma especie, por lo que se asume que la alteración producida por el arado afectó y posiblemente también ocasionó numerosas agresiones entre individuos de las 
colonias vecinas, al romperse el orden establecido por las pistas de forrageo, tal como se menciona con $M$. capitatus Latr. (Acosta et al., 1985). En la siguiente campaña, en 1998, se comprobó la desaparición en las calles de las especies $M$. bouvieri, $T$. nigerrima y $C$. velox, permaneciendo sólo $M$. barbarus y $C$. rosenhaueri, la última de las cuales ya no se encontró en el mes de Julio. La permanencia de $M$. barbarus en las calles despues del intenso arado se debe sin duda a la profundidad de los nidos de esta especie, más de 2 $\mathrm{m}$, mientras que los nidos de $C$. rosenhaueri suelen situarse entre los 0.5 y 1 metro (Plaza \& Tinaut, 1989).

Tabla 12. Número de nidos en el árbol $(n=60)$ en el olivar de Arenales. 1997 y 1998.

\begin{tabular}{cccccc} 
Año & Zona & \multicolumn{1}{c}{ Especie } & Mayo & Julio & Septiembre \\
\hline \multirow{2}{*}{1997} & Arenales & C. scutellaris & 3 & 5 & 1 \\
& & C. lateralis & 2 & 0 & 0 \\
\cline { 2 - 6 } & Total & 5 & 5 & 0 \\
\cline { 2 - 5 } 1998 & Arenales & C. scutellaris & 9 & 9 & 0 \\
& & C. lateralis & 2 & 1 & 0 \\
\cline { 2 - 5 } & & Total & 11 & 10 & 0 \\
\hline
\end{tabular}

Tabla 13. Valores medios, mínimos y máximos de nidos por árbol $(n=60)$ en Arenales. 1997 y 1998.

\begin{tabular}{|c|c|c|c|c|}
\hline \multirow[b]{2}{*}{ Año } & \multicolumn{4}{|c|}{ Número de nidos } \\
\hline & Mes & $x \pm s$ & Mín. & Máx. \\
\hline \multirow[t]{3}{*}{1997} & Мayo & $0.08 \pm 0.27 \mathrm{a} \mathrm{d}$ & 0.00 & 1.00 \\
\hline & Julio & $0.08 \pm 0.27 \mathrm{a}$ & 0.00 & 1.00 \\
\hline & Sept. & $0.01 \pm 0.12 \mathrm{a}$ & 0.00 & 1.00 \\
\hline \multirow[t]{3}{*}{1998} & Mayo & $0.20 \pm 0.40$ a d & 0.00 & 1.00 \\
\hline & Julio & $0.16 \pm 0.37 \mathrm{a}$ & 0.00 & 1.00 \\
\hline & Sept. & 0.00 & 0.00 & 0.00 \\
\hline
\end{tabular}

Las cifras con la misma letra no difieren significativamente entre si (Kruskal-Wallis, U de Mann Whitney, $\mathrm{P}>0.05)$. a: entre meses, $d$ : entre años.

\section{Número de nidos en el árbol}

El olivar de Arenales presentó dos especies con nidos en el árbol: Crematogaster scutellaris (Olivier) y Camponotus lateralis (Olivier). En los olivares Colomera 1 y 2 no se encontraron nidos de hormigas en los árboles, seguramente debido a la menor edad de éstos que no permitían el nicho especial que necesitan estas especies, según lo menciona CasevitzWeulersse (1972). El número de nidos permanece de Mayo a Julio, pero desciende en el mes de Septiembre sin diferencias significativas (1997: $\mathrm{P}=0.7$ y 1998: $\mathrm{P}=0.1)$. La especie $C$. scutellaris presentó el mayor número de nidos $(\mathrm{n}=3-9)$ durante la campaña, en cada año de estudio (Tablas 12 y 13). Morris (1997) menciona que fue el formícido más abundante en los dos olivares de Granada (Arenales y Parque de Invierno) y estuvo presente durante toda la temporada, es decir desde Abril hasta Septiembre. Esta especie, conocida en Andalucía Occidental como "morito" o "fraile", es una hormiga común en toda la región mediterránea y especialmente abundante en todos los alcornocales (Villagrán \& Ocete, 1990), pero también puede encontrarse en otros árboles: olivo, álamo, almendros e higueras (Casevitz-Weulersse, 1972). Soulié (1956) y Bonnemaison (1965) indican el carácter esencialmente arborícola de esta hormiga. Las obreras fueron observadas en troncos viejos cortados tanto a ras del suelo como a $0.80 \mathrm{~cm}$ de altura en donde la corteza estaba separada de la madera. La especie C. lateralis, con muy pocos nidos $(\mathrm{n}=1-2)$ (Tabla 12), es también citada por Morris (1997), como una de las menos frecuentes en el olivar.

\section{Agradecimientos}

El presente trabajo se ha realizado con el financiamiento de la Junta de Andalucía y la Beca de la AECI (Mutis) a I. Redolfi. Los autores agradecen a la Dra. Paqui Ruano y Dra. Consuelo Arellano las sugerencias en el análisis estadístico y la revisión del manuscrito; así como la asistencia técnica de $\mathrm{H}$. Barroso y A. Melgar.

\section{Literatura citada}

Acosta F.J. 1980. Las comunidades de hormigas en las etapas seriales del encinar. Tesis doctoral. Universidad Complutense de Madrid.

Acosta F.J., Ferradas M.A. \& Martin J.V. 1985. La competencia intraespecífica como causa de estabilidad en las rutas preferenciales de Messor capitatus (Latreille) (Hym. Formicidae). Studia Oecológica. VI: 267-278.

Adlung K.G. 1966. A critical evaluation of the european research on use of red wood ants (Formica rufa group) for the protection of forest against harmful insects. Dpto. Agricultural Research Service, Prís. 57: 167-189.

Andersen A.N. 1990. The use of ant communities to evaluate change: in Australian terrestrial ecosystem review and a recipe. Proc.Ecol. Soc. Aust. 16: 3-57.

Bernstein R.A. \& Gobbel M. 1979. Partitioning of space in communities of ants. J. Anim. Ecol. 48: 931-942.

Bonnemaison L. 1965. Enemigos animales de las plantas cultivadas y forestales. T III (Dípteros e Himenópteros). Ed. Occidente. Barcelona.

Buren W.F. \& Whitcomb W.H. 1977. Ants of citrus: some considerations. Proc. Int. Soc. Citriculture. 2: 523-558.

Casevitz-Weulersse J. 1972. Habitats et comportement nidificateur de Crematogaster scutellaris Olivier (Hym. Formicidae). Bull. Soc. ent. Fr. 77: 12-19.

Castro J., Campos P. \& Pastor M. 1996. Influencia de los sistemas de cultivo empleados en oliva $\mathrm{y}$ girasol sobre la composición de la fauna de 
artrópodos en el suelo. Bol. San. Veg. Plagas. 22: 557-570.

Cerda X. \& Retana J. 1988. Descripción de la comunidad de hormigas de un prado sabanoide en Canet de Mar (Barcelona). Ecología. (2): 333-341.

Cerda X. \& Retana J. 1998. Interference interacions and nest usurpation between two subordinate ant species. Oecologia. 113: 577-583.

Cerda X., Retana J., Bosch J. \& Alsina A.1989. Daily foraging activity and food collection of the thermophilic ant Cataglyphis cursor (Hym. Formicidae). Vie Milieu. 39: 207-212.

Cherix D. \& Rosengren R. 1979. Estimation de la fidelité sur pistes et de l'áge des forrageuses chez Formica lugubris Zett. dans le Jura suisse, par la methode de coloration au spray. C.R. UIEIS Sct. Francaise, Lausanne.

Cushman J.H., Martinsen G.D. \& Mazeroli A.I. 1988. Density and size dependent spacing of ant nests: evidence for intraspecific competition. Oecología. 77: 522-525.

David C.T. \& Wood D.E. 1980. Orientation to trails by a carpenter ant, Camponotus modoc (Hym. Formicidae) in a Grant sequoia forest. Can. Ent. 112: 993-1000.

Dejean A., Lenoir A. \& Godzinska E.J. 1994. The hunting behavior of Polyrhachis laboriosa, a nondominant arboreal ant of the African Equatorial Forest (Hymenoptera: Formicidae, Formicinae). Sociobiology. 30(1): 85-93.

Detrain C. 1990. Field study on foraging by the polymorphic ant species, Pheidole pallidula. Ins. Soc. 37(4): 315-332.

Fiala B., Grunsky H., Maschwitz U. \& Lisenmair K.E. 1994. Diversity of ant-plant interactions: protective efficacy in Macaranga species with different degrees of ant association. Oecologia. 97: 186-192.

Gonzalez R. 1989. Estudio bioecológico de Phloeotribus scarabaeoides (Bernard, 1788) (Coleoptera: Scolytidae) en la Provincia de Granada. Tesis Doctoral. Universidad de Granada.

Harrison J.S. \& Gentry J.B. 1981. Foraging pattern, colony distribution and foraging range of the Florida harvester ant Pogonomyrmex badius. Ecology. 62 (6): 1467-1473.

Jacson, D.A. 1984. Ant distribution patterns in Cameroonian cocoa plantation: investigation of the ant mosaic hypothesis. Oecologia (Berl.). 62: 318324.

Leston D. 1978. A neotropical ant mosaic. Ann. Entomol. Soc. Am. 71: 649-53.

Majer J.D. 1972. The ant mosaic in Ghana cocoa farms. Bull. Ent. Res. 62: 151-160.

Majer J.D. 1987. Comparison of the arboreal ant mosaic in Ghana, Brazil, Papua New Guinea and Australia its structure and influence on arthropod diversity. :115-141. In Hymenoptera and
Biodiversity. Eds. La Salle, Gauld. C.A.B. International, Wallingford, UK.

Majer J.D. 1994. Introduction of ants as potential biological control agents, with particular reference to cocoa. Harvest. 16(1,2): 1-4.

Majer J.D. \& Beeston G. 1996. The Biodiversity Integrity Index: An Ilustration Using ants in Western Australia. Cons. Biol. 10(1): 65-73.

Majer J.D. \& Delabie J.H.C. 1993. An evaluation of Brasilian cocoa farm ants as potential biological control agents. Journal of Plant Protection in the Tropics. 10(1): 43-49.

Morris T.I. 1997. Interrelaciones entre olivos, plagas y depredadores. Tesis Doctoral. Universidad de Granada. España.

Morris T.I., Campos M., Jervis M.A., McEwen P.K. \& Kidd N.A.C. 1998a. Potential effects of various ant species on green lacewing, Chrysoperla carnea (Stephens) (Neuropt., Chrysopidae) egg numbers. J. Appl. Ent. 122: 401-403.

Morris T.I., Symondson W.O.C., Kidd N.A.C., Jervis M.A. \& Campos M. 1998b. Are ants significant predator of the olive moth, Prays oleae?. Crop Protection. 17(4): 365-366.

Morris T.I., Symondson W.O.C., Kidd N.A.C. \& Campos M. 2002. The effect of different ant species on the olive moth, Prays oleae (Bern.), in Spanish olive orchard. J. Appl. Ent. 126: 1-6.

Ofer Y., Harris Y, Beiles A. \& Nevo E. 1996. Biodiversity of ants at "Evolution canyon", Nahal Oren, MT. Carmel, Israel. Israel Journal Entomology. 30: 115-119.

Orr A.G. \& Charles J.K. 1994. Foraging in the giant forest ant, Camponotus gigas (Smith) (Hymenoptera: Formicidae): evidence for temporal and spatial specialization in foraging activity. Journal of Natural History. 28: 861-872.

Oster G.F. \& Wilson E.O. 1978. Caste and Ecology in the Social Insects. Princeton Univ. Press. Princeton.

Paulson G.S. \& Akre R.D. 1992. Evaluating the effectiveness of ants as biological control agents of pear Psylla (Homoptera: Psyllidae). J. Econ. Ent. 81(1): 70-73.

Peng R.K., Chistian K. \& Gibb K. 1997. Distribution of the green ant, Oecophylla samaragdina (F.) ( Hymenoptera: Formicidae), in relation tu native vegetation and the insect pests in cashew plantations in Australia. International Journal of Pest Management. 43(3): 203-211.

Pérez P. \& Prieto P. 1980. C.S.I.C. Memoria explicativa de los mapas de suelos y vegetación de la provincia de Granada.

Perfecto I. \& Snelling R. 1995. Biodiversity and the transformation of a tropical agroecosystem: ants in coffee plantations. Ecol. Appl. 5(4): 1084-1097.

Plaza J. \& Tinaut A. 1989. Descripción de los hormigueros de Cataglyphis rosenhaueri (Emery, 
1906) y Cataglyphis iberica (Emery, 1906) en diferentes biotopos de la provincia de Granada (Hym. Formicidae). Boln. Asoc. esp. Entom. 13: 109-116.

Porter S.D. \& Jorgensen C.D. 1981. Foragers of the Harvester ant Pogonomyrmex owyheei: A disposable Caste?. Behav. Ecol. Sociobiol. 9: 247256.

Rabitsch W.B. 1997. Tissue-specific accumulation patterns of $\mathrm{Pb}, \mathrm{Cd}, \mathrm{Cu}, \mathrm{Zn}, \mathrm{Fe}$, and $\mathrm{Mn}$ in workers of three ant species (Formicidae, Hymenoptera) from a metal-polluted site. Arch. Environ. Contam. Toxicol. 32: 172-177.

Redolfi I. 1999. Las hormigas (Hymenoptera: Formicidae) en el agroecosistema del olivo en Granada, España. Tesis Doctoral. Universidad de Granada. España.

Redolfi I., Tinaut A., Pascual F. \& Campos M. 1999. Qualitative aspects of myrmecocenosis (Hym.,Formicidae) in olive orchards with different agricultural management in Spain. J. Appl. Ent. 123: 621-627.

Redolfi I., Tinaut A., Pascual F. \& Campos M. 2003. Patrón de actividad de Tapinoma nigerrima (Nylander) y Crematogaster scutellaris (Olivier) (Hymenoptera, Formicidae) en el cultivo de olivo y en el laboratorio. Zool. baetica. 13/14: 37-55.
Rivas-Martinez S. 1987. Memoria del mapa de series de vegetación de España. Ed. ICONA, Madrid.

Room P.M. 1971. The relative distribution of ant species on Ghana's cocoa farms. Journal of Animal Ecology. 40: 735-751.

Room P.M. 1974. Diversity and organisation of ground foraging ant faunas of forest, grasslands and tree crops in Papua New Guinea. Aust. J. Zool. 23(1): 34-37.

Rosengren R. 1977. Foraging strategy of wood ants (Formica rufa group). I Age polyethism and topografhic traditions. Act. Zool. Fenn. 149: 1-30.

Soulie J. 1956. La nidificación chez les espéces francaises du genere Crematogaster Lund (Hym. Formicoidea). Ins. Soc. III (1): 93-105.

Tinaut A. 1979. Estudio de la mirmecofauna de los Borreguiles de S. Juan (Sierra Nevada, Granada) (Hym. Formicidae). Bol. Asoc. Esp. Entom. 3: 173-183.

Weseloh R.M. 1993. Maipulation of Forest Ant (Hymenoptera: Formicidae) abundance and resulting impact on Gypsy Moth (Lepidoptera: Lymantriidae) populations. Env. Ent. 22(3): 587594.

Weseloh R.M. 1996. Effect of supplemental foods on foraging behavior of forest ants in Connecticut. Env. Ent. 25 (4): 848-853.

\footnotetext{
${ }^{1}$ Estación Experimental del Zaidín (CSIC), Profesor Albareda 1, Granada, España (1996-1999).Universidad Nacional Agraria La Molina. Facultad de Ciencias. Departamento Académico de Biología. Laboratorio de Control Biológico y Ecología de Artrópodos. Av. La Molina s/n. La Molina. Apartado 12-056. Lima12. Perú. Correo electrónico: rhi@lamolina.edu.pe.

${ }^{2}$ Universidad de Granada, Campus Universitario Fuentenueva. 18071. Granada. España.

Correos electrónicos: hormiga@ugr.es/ fpascual@ugr.es.

${ }^{3}$ Estación Experimental del Zaidín (CSIC).Correo electrónico: mcampos@eez.csic.es.
} 